\title{
Efficient ultraviolet electroluminescence from a Gd-implanted silicon metal-oxide-semiconductor device
}

\author{
J. M. Sun, ${ }^{\text {a) }}$ W. Skorupa, T. Dekorsy, and M. Helm \\ Institute of Ion Beam Physics and Materials Research, Forschungszentrum Rossendorf, POB 510119 , \\ D-01314 Dresden, Germany \\ L. Rebohle and T. Gebel \\ Nanoparc GmbH, Dresden, Germany
}

\begin{abstract}
Strong ultraviolet electroluminescence with an external quantum efficiency above $1 \%$ is observed from an indium-tin oxide/ $\mathrm{SiO}_{2}: \mathrm{Gd} / \mathrm{Si}$ metal-oxide-semiconductor structure. The $\mathrm{SiO}_{2}: \mathrm{Gd}$ active layer is prepared by thermal oxidation followed by $\mathrm{Gd}^{+}$implantation and annealing. The electroluminescence spectra show a sharp peak at $316 \mathrm{~nm}$ from the ${ }^{6} P_{7 / 2}$ to ${ }^{8} S_{7 / 2}$ transition of $\mathrm{Gd}^{3+}$ ions. Micrometer-sized electroluminescent devices are demonstrated.
\end{abstract}

Light sources operating in the ultraviolet (UV) region are required for a number of applications, including solidstate lighting, biological agent detection, sterilization, and covert communication. Strong UV light emission from AlGaN-based emitters has been reported emitting in the UV range from 265 to $325 \mathrm{~nm} .{ }^{1-3}$ However, for the development of microlight sources for on-chip analysis, such as biochips, biosensors, microflorescent displays, etc., efficient siliconbased microlight sources are required which can be integrated into silicon integrated circuits. Silicon-based UV light emitters are promising for such applications. Recently, light emitters incorporating rare-earth $\mathrm{Er}^{3+}, \mathrm{Tm}^{3+}, \mathrm{Tb}^{3+}$, and $\mathrm{Ce}^{3+}$-doped $\mathrm{Si}$-rich $\mathrm{SiO}_{2}$ have been reported with emission in the infrared to blue-green spectral regions. ${ }^{4,5}$ Strong blueviolet emission at $390 \mathrm{~nm}$ has been reported in $\mathrm{Ge}^{3+}$-implanted $\mathrm{SiO}_{2}$ metal-oxide-semiconductor (MOS) structures. ${ }^{6}$ Efficient silicon-based UV light emitters at a shorter wavelength from silicon device have not yet been reported for the time being. In this letter, we demonstrate a silicon-based efficient UV light emitter based on a $\mathrm{SiO}_{2}: \mathrm{Gd}$ MOS structure delivering a sharp emission peak at $316 \mathrm{~nm}$ from $\mathrm{Gd}^{3+}$ ions, which is comparable to the efficient UV emission of $\mathrm{ZnF}_{2}: \mathrm{Gd}^{7}$ External quantum efficiencies above $1 \%$ are achieved with not fully optimized devices. Device sizes below $1 \mu \mathrm{m}$ can be easily achieved with standard complementary MOS (CMOS) technology.

Electroluminescent (EL) devices are prepared by standard silicon CMOS technology on 4 in. $n$-type silicon wafer with resistivity of $2-5 \Omega \mathrm{cm}$. The structure consists of an active gate oxide area surrounded by a field oxide $(1 \mu \mathrm{m}$ thick). The active layer is a $100 \mathrm{~nm}$ thick thermally grown $\mathrm{SiO}_{2}$ layer implanted with $\mathrm{Gd}^{+}$ions at two energies of 50 and $110 \mathrm{keV}$ with doses of $5 \times 10^{14}$ and $1 \times 10^{15} \mathrm{~cm}^{-2}$, respectively. After annealing at $800^{\circ} \mathrm{C}$ for $1 \mathrm{~h}$, a plasma treatment was performed in a mixture of oxygen/hydrogen (90/10) for $5 \mathrm{~min}$. The gate electrode consists of a $100 \mathrm{~nm}$ thick indiumtin oxide (ITO) deposited by rf sputtering. Various shapes of MOS structures with different feature sizes in the range of 1

${ }^{\text {a) }}$ Author to whom correspondence should be addressed; electronic mail: j.sun@fz-rossendorf.de to $500 \mu \mathrm{m}$ were fabricated for testing the function of the EL devices dependent upon the geometry.

EL spectra were measured on a MOS structure with $500 \mu \mathrm{m}$ diameter with a constant current supplied by a sourcemeter (Keithley 2410). EL signals were recorded at room temperature with a monochromator and a photomultiplier. The absolute EL power from the device was measured using a calibrated optical power meter. The external EL power efficiency is calculated by integrating the total EL output power from the front surface. Photoluminescence (PL) and photoluminescence excitation (PLE) spectra were also measured with the same system using UV excitation from a $75 \mathrm{~W}$ Xe lamp.

Figure 1 shows the PL and PLE spectra from the $\mathrm{SiO}_{2}$ : Gd gate oxide without an ITO electrode on top. The PL spectrum under the excitation of $195 \mathrm{~nm}$ UV light shows a sharp peak at $316 \mathrm{~nm}$ arising from the transition of ${ }^{6} P_{7 / 2}$ to ${ }^{8} S_{7 / 2}$ of the $\mathrm{Gd}^{3+}$ ions. ${ }^{8}$ The PLE spectrum of the $316 \mathrm{~nm}$ emission exhibits two excitation peaks at 195 and $274 \mathrm{~nm}$. The peak at $195 \mathrm{~nm}$ corresponds to the excitation from ${ }^{8} S_{7 / 2}$ to ${ }^{6} G_{13 / 2}$ and the peak at $274 \mathrm{~nm}$ can be assigned to the ${ }^{8} S_{7 / 2}$ to ${ }^{6} I_{J}$ excitation of $\mathrm{Gd}^{3+}$ (Ref. 9).

Figure 2 shows the EL spectra of the $\mathrm{SiO}_{2}: \mathrm{Gd}$ at an injection current of $100 \mu \mathrm{A}$ for the MOS structure with a

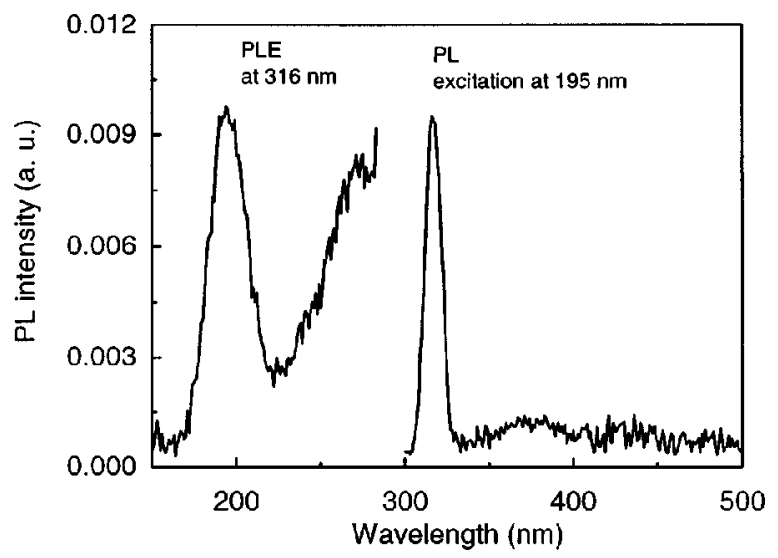

FIG. 1. PL and PLE spectra from the $100 \mathrm{~nm} \mathrm{SiO}_{2}$ : Gd layer. The PL was excited by $195 \mathrm{~nm}$ UV light, and the PLE was measured with a detection wavelength of $316 \mathrm{~nm}$. 


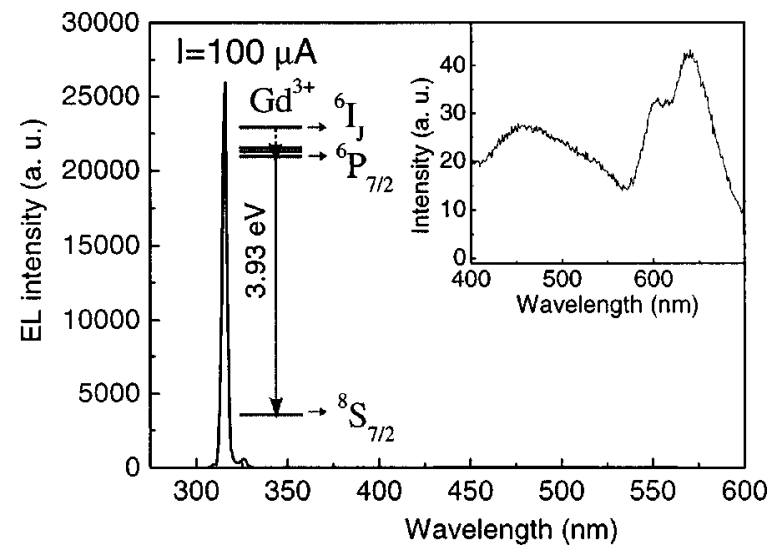

FIG. 2. EL spectrum of the $\mathrm{SiO}_{2}$ : Gd MOS device at $100 \mu \mathrm{A}$ current and a sketch of the involved electronic transition. The inset shows the emission in the $400 \mathrm{~nm}$ to $700 \mathrm{~nm}$ region. Note that the intensity in this region is nearly three orders of magnitude lower than the emission at $316 \mathrm{~nm}$.

diameter of $500 \mu \mathrm{m}$. The spectra show a strong peak at $316 \mathrm{~nm}$ from the transition of the lowest excited states of ${ }^{6} P_{7 / 2}$ to ${ }^{8} S_{7 / 2}$ from the $\mathrm{Gd}^{3+}$ ions. Two broad peaks in the visible range centered at $460 \mathrm{~nm}$ and $650 \mathrm{~nm}$ are observed at higher current injection, as shown in the inset of Fig. 2. These bands have an intensity about three orders of magnitude lower than the $316 \mathrm{~nm}$ peak at the same injection current. The bands originate from neutral oxygen vacancies and nonbridging oxygen hole centers as typical network defects of the amorphous silicon dioxide created by the implantation process. ${ }^{10,11}$

In order to clarify the excitation process of the UV emission, we analyze the current-field characteristics of the device as shown in Fig. 3. The ratio of the injection current density and the square of the electric field, i.e., $J / E^{2}$, plotted versus the reciprocal of the electric field which allows us to verify Fowler-Nordheim $(\mathrm{F}-\mathrm{N})$ tunneling injection of hot electrons at $\mathrm{SiO}_{2} / \mathrm{Si}$ interface in the MOS structure expressed as $J / E^{2}=A \exp (-B / E), A$ and $B$ are constants. ${ }^{12}$ This expression is valid in the range from $8.5 \mathrm{MV} / \mathrm{cm}$ to $10.3 \mathrm{MV} / \mathrm{cm}$. The inset of Fig. 3 shows the EL intensity of the $316 \mathrm{~nm}$ peaks from $\mathrm{Gd}^{3+}$ as a function of the injected current of the MOS device. An external quantum efficiency of $1 \%$ is obtained for this device (with a power efficiency of $\left.4-6 \times 10^{-4}\right)$. The threshold electric field for detection of the $\mathrm{UV}$ is about $8.5 \mathrm{MV} / \mathrm{cm}$, which is the same as the threshold electric field for $\mathrm{F}-\mathrm{N}$ tunneling injection of the hot electrons into the conduction band of the $\mathrm{SiO}_{2}$ for strong electric heat-

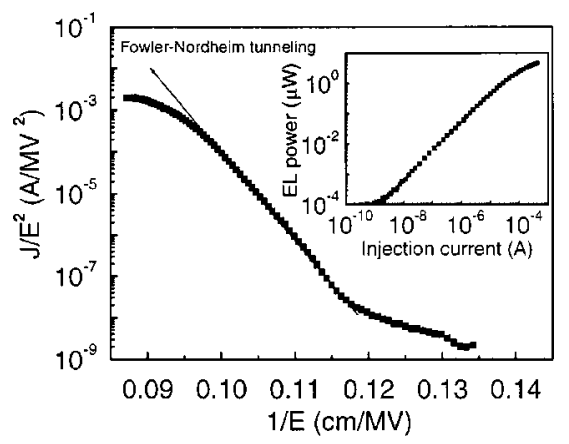

FIG. 3. The current density of the $\mathrm{SiO}_{2}$ : Gd MOS device divided by the squared electric field is plotted vs the reciprocal electric field ( $\mathrm{F}-\mathrm{N}$ plot of the current-field characteristic). The inset is the integrated EL power vs the injection current.
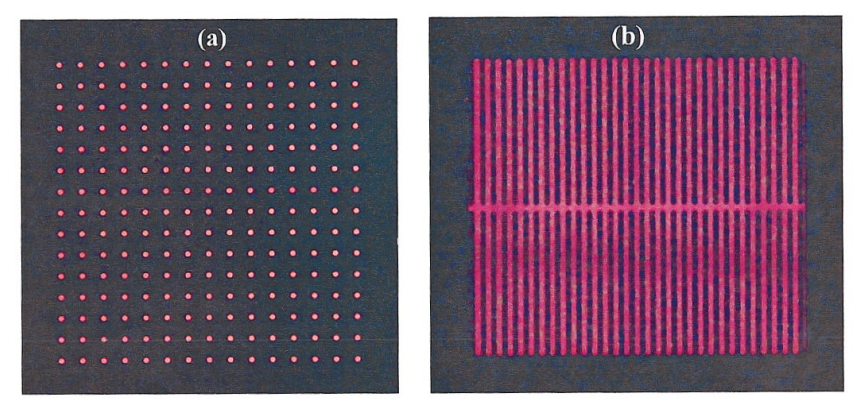

FIG. 4. (Color) CCD photograph of the EL from two different structures: (a) matrix of $15 \times 15$ dots with a diameter of $20 \mu \mathrm{m}$ and spacing of $70 \mu \mathrm{m}$ and (b) comblike structure with an EL strip width of $4 \mu \mathrm{m}$. Note the observed visible light is 2000 times weaker than the UV peaks, which is not observed by the CCD camera.

ing. According to the study of DiMaria et al. ${ }^{13}$ under such high electric fields of $8.5-11 \mathrm{MV} / \mathrm{cm}$, as is necessary for the operation of our devices, the average hot electron energy is above $4 \mathrm{eV}$ with respect to the bottom of the conductionband edge of $\mathrm{SiO}_{2}$; the average energy of the hot electrons is larger than the EL photon energy of $3.93 \mathrm{eV}$. Hence, a large number of hot electrons are capable of generating efficiently UV light from $\mathrm{Gd}^{3+}$ by impact excitation in the $\mathrm{SiO}_{2}$ host.

Figure 4 shows a matrix of $15 \times 15$ devices of a $20 \mu \mathrm{m}$ diameter MOS structure operated with a total injection current of $100 \mu \mathrm{A}$ and a high-resolution comblike structure with EL strips of $4 \mu \mathrm{m}$ width, both imaged with a Si charge coupled device (CCD) camera under an optical microscope. Note that only the visible part of the spectrum from the $\mathrm{SiO}_{2}$ defect EL is observed, which is nearly three orders of magnitude weaker than the UV light. The smallest feature size is $2 \mu \mathrm{m}$ which is limited by the capability of our lithography system. However, smaller sizes below $1 \mu \mathrm{m}$ are possible for high-resolution display or biochip applications. Combination with red-green-blue phosphors, efficient silicon-based UV light emitters can be better candidates for the fabrication of high-resolution full-color microdisplays as compared to $\mathrm{ZnF}_{2}: \mathrm{Gd}$ thin-film EL devices, ${ }^{7}$ since the fabrication of silicon-based devices is fully compatible with standard MOS technology.

In summary, strong UV EL with an external quantum efficiency above $1 \%$ was reported from an $\mathrm{ITO} / \mathrm{SiO}_{2}: \mathrm{Gd} / \mathrm{Si}$ MOS structure. The UV is generated by impact excitation of $\mathrm{Gd}^{3+}$ ions by hot electrons. External quantum efficiencies above $1 \%$ were obtained at room temperature for further optimization by changing the oxide thickness and Gd concentration. EL devices on a micrometer-scale can be fabricated employing standard CMOS technology.

The authors would like to thank I. Winkler and J. Schneider for the ion implantation, and H. Felsmann, C. Neisser, and G. Schnabel for the processing of the MOS structures.

${ }^{1}$ A. J. Fischer, A. A. Allerman, M. H. Crawford, K. H. A. Bogart, S. R. Lee, R. J. Kaplar, W. W. Chow, S. R. Kurtz, K. W. Fullmer, and J. J. Figiel, Appl. Phys. Lett. 84, 3394 (2004).

${ }^{2}$ A. Yasan, R. McClintock, K. Mayes, D. Shiell, L. Gautero, S. R. Darvish, P. Kung, and M. Razeghi, Appl. Phys. Lett. 83, 4701 (2003).

${ }^{3}$ A. Chitnis, J. P. Zhang, V. Adivarahan, M. Shatalov, S. Wu, R. Pachipulusu, V. Mandavilli, and M. A. Khan, Appl. Phys. Lett. 82, 2565 (2003).

${ }^{4}$ M. E. Castagna, S. Coffa, M. Monaco, A. Muscara, L. Caristia, S. Lorenti, and A. Messina, Mater. Res. Soc. Symp. Proc. 770, 12.1.1 (2003).

${ }^{5}$ S. Y. Seo, J. H. Shin, B. S. Bae, N. Park, J. J. Penninkhof, and A. Polman, 
Appl. Phys. Lett. 82, 3445 (2003).

${ }^{6}$ L. Rebohle, J. von Borany, R. A. Yankov, W. Skorupa, I. E. Tyschenko, H. Fröb, and K. Leo, Appl. Phys. Lett. 71, 2809 (1997).

${ }^{7}$ T. Senda, Y.-J. Cho, Takashi Hirakawa, H. Okamoto, H. Takakura, and Y. Hamakawa, Jpn. J. Appl. Phys., Part 1 39, 4716 (2000).

${ }^{8}$ N. Miura, T. Sasaki, and H. Matsumoto, Jpn. J. Appl. Phys., Part 2 30, L1815 (1991).

${ }^{9}$ R. T. Wegh, H. Donker, A. Meijerink, R. J. Lamminmäki, and J. Hölsä, Phys. Rev. B 56, 13841 (1997).
${ }^{10}$ L. Rebhole, J. von Borany, H. Fröb, and W. Skorupa, Appl. Phys. B: Lasers Opt. 70, 1 (2000).

${ }^{11}$ A. N. Trukhin, M. Goldberg, J. Jansons, H.-J. Fitting, and I. A. Tale, J. Non-Cryst. Solids 223, 114 (1998).

${ }^{12}$ J. A. Lopez-Villanueva, J. A. Jimenez-Tejada, P. Cartujo, J. Bausells, and J. E. Carceller, J. Appl. Phys. 70, 3712 (1991).

${ }^{13}$ D. J. DiMaria, T. N. Theis, J. R. Kirtley, F. L. Pesavento, D. W. Dong, and S. D. Brorson, J. Appl. Phys. 57, 1214 (1985). 